\title{
Evaluation of functional connectivity in subdivisions of the thalamus in schizophrenia
}

Jinnan Gong, Cheng Luo, Xiangkui Li, Sisi Jiang, Budhachandra S. Khundrakpam, Mingjun Duan, Xi Chen and Dezhong YaO

\section{Background}

Previous studies in schizophrenia revealed abnormalities in the cortico-cerebellar-thalamo-cortical circuit (CCTCC) pathway, suggesting the necessity for defining thalamic subdivisions in understanding alterations of brain connectivity.

\section{Aims}

To parcellate the thalamus into several subdivisions using a datadriven method, and to evaluate the role of each subdivision in the alterations of CCTCC functional connectivity in patients with schizophrenia.

\section{Method}

There were 54 patients with schizophrenia and 42 healthy controls included in this study. First, the thalamic structural and functional connections computed, based on diffusion magnetic resonance imaging (MRI, white matter tractography) and restingstate functional MRI, were clustered to parcellate thalamus. Next, functional connectivity of each thalamus subdivision was investigated, and the alterations in thalamic functional connectivity for patients with schizophrenia were inspected.

\section{Results}

Based on the data-driven parcellation method, six thalamic subdivisions were defined. Loss of connectivity was observed between several thalamic subdivisions (superior-anterior, ventromedial and dorsolateral part of the thalamus) and the sensorimotor system, anterior cingulate cortex and cerebellum in patients with schizophrenia. A gradual pattern of dysconnectivity was observed across the thalamic subdivisions.

Additionally, the altered connectivity negatively correlated with symptom scores and duration of illness in individuals with schizophrenia.

\section{Conclusions}

The findings of the study revealed a wide range of thalamic functional dysconnectivity in the CCTCC pathway, increasing our understanding of the relationship between the CCTCC pathway and symptoms associated with schizophrenia, and further indicating a potential alteration pattern in the thalamic nuclei in people with schizophrenia.

\section{Declaration of interest}

None.

\section{Keywords}

Schizophrenia; thalamus; functional connectivity; parcellation; tractography.

\section{Copyright and usage}

(c) The Royal College of Psychiatrists 2019.
The thalamus relays information from the cerebellum, subcortical nuclei and neocortex, ${ }^{1}$ and thus plays a key role in information processing in brain. Altered thalamus and thalamo-cortical pathways have recently been revealed in patients with schizophrenia., ${ }^{2,3}$ Although altered cortico-cerebellar connectivity has been shown in schizophrenia (reviewed by Lungu $e t a l^{4}$ ), it is unclear whether the alterations are caused by the cerebellum or the abnormality of the thalamic relaying. Thus, systematic study of the thalamic connections, particularly subtle examination of the thalamic subdivisions, will be helpful in exploring the pathophysiology of schizophrenia. Most functional MRI (fMRI) studies investigated thalamic functional features in schizophrenia using whole thalamus as the region of interest (ROI). However, it is difficult to identify whether the alterations of the thalamo-cortical functional connectivity in schizophrenia are due to a specific region or the entire thalamus, because the composition of thalamus is complex. A connectivity-based parcellation approach makes it possible to segment brain regions in vivo according to the connectivity pattern of diffusion MRI (dMRI) or fMRI data. ${ }^{5}$ Recently, a few studies focused on the functional connectivity of thalamic subdivisions such as thalamic segmentation (thalamic prefrontal subdivisions, thalamic motor subdivisions and so on) according to its functional connectivity, ${ }^{6}$ providing more information about the alterations in the thalamo-cortical coupling in schizophrenia.

A method combining white matter connectivity and restingstate functional connectivity (rsFC) to divide the thalamus into several subdivisions was proposed recently. ${ }^{7}$ This approach efficiently integrated information from structural and functional connectivity within a participant group to provide a flexible division scheme. ${ }^{8}$ Similar to this approach, the current study combined dMRI and fMRI data to parcellate the thalamus. Next, differences in the rsFC maps of the subthalamic divisions between patients with schizophrenia and healthy controls were obtained. Finally, the relationships between functional alterations and clinical symptoms were evaluated in patients with schizophrenia.

\section{Method}

\section{Data-set and demographics}

A total of 54 patients with schizophrenia, who were diagnosed using the structured clinical interview for DSM-IV Axis I disorders clinical version (SCID-I-CV), ${ }^{9}$ were recruited from in-patient and out-patient treatment facilities in the Clinical Hospital of Chengdu Brain Science Institute. Each patient completed the Positive and Negative Syndrome Scale (PANSS) ${ }^{10}$ during a semistructured interview to assess psychotic symptoms. All patients took medication at the time of the study. The information about usage of antipsychotic medication was collected from the patients. To evaluate the effects of various antipsychotic drugs, we used a standardised quantitative formula for each antipsychotic medication so as to determine the equivalent for chlorpromazine. ${ }^{11}$ The mean chlorpromazine equivalent (CPE) dose of the antipsychotics was $281.62 \mathrm{mg}$ per day (s.d. $=122.72$, Table 1 ). We recruited 42 healthy controls matched for age, gender and years of education. 


\begin{tabular}{|c|c|c|c|c|}
\hline & \multirow{2}{*}{$\begin{array}{l}\text { Control group } \\
(n=42)\end{array}$} & \multirow{2}{*}{$\begin{array}{l}\text { Schizophrenia group } \\
(n=54)\end{array}$} & \multicolumn{2}{|c|}{ Statistics } \\
\hline & & & $t$ or $\chi^{2}$ & $P$ \\
\hline Gender, men/women & $24 / 18$ & $34 / 20$ & 0.335 & 0.563 \\
\hline Mean FD/FR (mm/degree) & $0.044 / 5.041 \times 10^{-4}$ & $0.044 / 5.741 \times 10^{-4}$ & $0.04 / 1.3$ & $0.97 / 0.20$ \\
\hline Antipsychotic medicated, yes: \% & - & 100 & - & - \\
\hline Age, mean (s.d.) & $39.6(11.8)$ & $38.1(12.6)$ & 0.582 & 0.562 \\
\hline Duration of illness, years: mean (s.d.) & - & $13.9(10.6)$ & - & - \\
\hline Education, years: mean (s.d.) & $10.9(3.1)$ & $11.3(2.7)$ & 0.976 & 0.332 \\
\hline \multicolumn{5}{|l|}{ Positive and Negative Syndrome Scale, mean (s.d.) } \\
\hline Positive & - & $14.0(5.9)$ & - & - \\
\hline Negative & - & $20.7(6.2)$ & - & - \\
\hline General & - & $28.5(6.1)$ & - & - \\
\hline Auditory Hallucination Rating Scale, ${ }^{a}$ mean (s.d.) & - & $9.1(11.4)$ & - & - \\
\hline Chlorpromazine equivalent dose, $\mathrm{mg} / \mathrm{day}^{\mathrm{b}}$ mean (s.d.) & - & $281.62(122.72)$ & - & - \\
\hline
\end{tabular}

All participants had no neurological illness, traumatic brain injury or substance-related disorders.

This study was approved by the Ethics Committee of the Clinical Hospital of Chengdu Brain Science Institute in accordance with the Declaration of Helsinki. Before MRI scanning, all participants provided written informed consent.

\section{Magnetic resonance imaging parameters}

All participants were scanned using a 3.0 Tesla MRI scanner (GE Discovery MR 750, USA), at the MRI Center of University of Electronic Science and Technology of China. Specifically, restingstate fMRI data was acquired with axial slices parallel to the anterior-posterior commissure using a standard $\mathrm{T}_{2}{ }^{*}$-weighted echo planar imaging (EPI) pulse sequence (repetition time (TR)/echo time $(\mathrm{TE})=2000 \mathrm{~ms} / 30 \mathrm{~ms}$, flip angle $90^{\circ}$, field of view $(\mathrm{FOV})=$ $24 \times 24 \mathrm{~cm}$, acquisition matrix $64 \times 64$, slice number 35 , scanned interleaved and slice thickness $4 \mathrm{~mm}$ with no gap) covering the whole brain. In total, 255 volumes were obtained in $8.5 \mathrm{~min}$ for each participants (35 slices per volume). Structural MRI data were obtained using a $\mathrm{T}_{1}$-weighted 3D FSPGR image (FOV $=25.6 \times$ $25.6 \mathrm{~cm}$, flip angle $9^{\circ}, 158$ slices per volume) through the brain with $1 \times 1 \times 1 \mathrm{~mm}$ voxel size.

After obtaining three unweighted images, diffusion-weighted images were acquired in 64 directions using a diffusion-weighted spinecho EPI sequence with the following parameters: $b=1000 \mathrm{~s} / \mathrm{mm}^{2}$, $\mathrm{TR}=8500 \mathrm{~ms}$, frequency direction right/left, acquisition matrix $128 \times 128, \quad$ FOV $=25.6 \times 25.6 \mathrm{~cm}$, slice thickness $2 \mathrm{~mm}$ and 78 slices. In addition, another three unweighted images were obtained in the anterior/posterior frequency direction to estimate and correct for susceptibility-induced distortions.

\section{Parcellation of the thalamus}

After preprocessing of dMRI and fMRI data (see supplementary Data Preprocessing available at https://doi.org/10.1192/bjp.2018.299 for details), a two-step pipeline was introduced to parcellate the thalamus into several subdivisions by using the combined information of dMRI and fMRI. ${ }^{7}$ Briefly, the first step is voxel-based probabilistic tractography seeding from the thalamic voxels. Structurally weighted subregions (i.e. the 'thalamic origins' of these tractograms) are identified by independent component analysis (ICA) of these tractograms. Then, the second step is parcellation of the thalamus according to the clustering results ${ }^{13}$ of functional connectivity with seeds at these structurally defined thalamic origins (for details, see supplementary Data 1). Thus, the thalamic subdivisions generated by this method are deemed to have similar structural and functional connectivity patterns. Subsequently, these thalamic subdivisions were used as seeds to acquire their functional connectivity maps.

\section{Functional connectivity evaluation of the thalamic subdivisions}

The RsFC map of each thalamic subdivision was computed using Pearson's correlation between the time series of the thalamic subdivision and the whole brain for each participant. Subsequently, a second-level multiple regression was used to test the differences in rsFC maps between groups. Age and gender were used as covariates, and Bonferroni correction for multiple comparisons was performed for the voxel-level analyses.

\section{Correlation of clinical features and neuroimaging}

Spearman's rank correlation coefficient was used to quantify the relationship between clinical features including positive/negative symptom scales (PANSS) and duration of illness, and neuroimaging metrics (the functional connectivity value of each voxel that showed a significant intergroup difference). In addition, in order to investigate the effect of antipsychotic medication usage on thalamic functional connectivity, Pearson's correlation coefficient was calculated between $\mathrm{CPE}$ and the functional connectivity value of each voxel with significant intergroup difference.

\section{Results}

\section{Participants}

Gender, age, and education level did not differ between the schizophrenia and control groups $(P>0.05$, Table 1$)$. There was also no group difference in the level of head motion $(P>0.05$, supplementary Fig. 1).

\section{Parcellation of the thalamus}

All voxel-based tractography maps were decomposed to 29 spatial components for the left thalamus and 26 for the right thalamus using tensor-ICA. This information was used to define 29- and 26-weighted thalamic origins for the left and right thalamus, respectively. Depending on the clustering process of functional connectivity maps for the 55 weighted thalamic origins, they were labelled into six sets (supplementary Fig. 2). Subsequently, the bilateral thalamus was parcellated into six nearly symmetrical subdivisions. These were designated as the ventroposterior part 
(ROI 1), the ventral postmedian part (ROI 2), the dorsomedial part (ROI 3), the ventromedial part (ROI 4), the dorsolateral part (ROI 5) and the ventral anterior part (ROI 6) of the thalamus. Supplementary Table 1 shows the composition of the thalamic nuclei for each subdivision according to the Morel thalamic atlas.

\section{Functional dysconnectivity of the thalamic subdivisions' loop}

We found that the thalamo-cortical rsFC maps that exhibited negative connectivity in the control group were decreased in the schizophrenia group including thalamic functional connectivity in the sensorimotor network (namely the precentral and postcentral gyri, known as SM1), visual cognition network (namely the lingual gyrus, cuneus and precuneus) and auditory cognition network (i.e. the superior, middle and inferior temporal gyrus). However, specific differences were observed among functional connectivity maps for each thalamic subdivision.

In detail, functional connectivity in schizophrenia was decreased between ROI 3 (Fig.1 and supplementary Table 2) and the visual cognition network, including the left calcarine, the right lingual gyrus and the precuneus. In functional connectivity comparison of ROI 4 (Fig. 2(a) and supplementary Table 2), ROI 5 (Fig. 2(b) and supplementary Table 2) and ROI 6 (Fig. 2(c) and supplementary Table 3), patients exhibited lower functional connectivity values in the SM1, occipital and temporal regions. In addition, loss of positive interaction was observed between anterior cingulate cortex (ACC) and ROI 4. Moreover, the connectivity between insula and thalamic subdivisions (including ROI 5 and ROI 6) showed an increased pattern in the schizophrenia group, whereas the control group showed negative interaction.

Compared with the control group, reduced thalamo-cerebellar positive interaction was also observed in patients for each thalamic subdivision (supplementary Fig. 3). Additionally, the intrathalamic positive interaction was decreased in the schizophrenia group (supplementary Fig. 4) including ROI 2, ROI 4, ROI 5 and ROI 6 (supplementary Table 4).

\section{Correlation of clinical features and neuroimaging}

Functional connectivity between the right precentral gyrus and ROI 5 positively correlated with the total PANSS score, the general psychopathology scale $(P<0.001$, Fig. 3$)$ and the negative syndrome scale in the schizophrenia group $(P<0.001)$. Functional connectivity between ROI 5 and the left superior temporal gyrus and the right lingual gyrus also positively correlated with severity of the negative symptoms of the schizophrenia group $(P<0.001)$. Additionally, the intrathalamic interaction in ROI 6 negatively correlated with the negative symptoms of those in the schizophrenia group $(P<0.001)$.

The intrathalamic functional integration of ROI 4 and ROI 5 negatively correlated with the duration of disease $(P<0.001$, supplementary Fig. 5). Some regions in the functional connectivity map of ROI 5 correlated with CPE (supplementary Fig. 6). For example, the functional connectivity value in the right crus I and II of cerebellum negatively correlated with CPE $(P<0.005)$. Functional connectivity values in the right lateral occipital cortex (LOC), the inferior division $(P<0.001)$ and in the right primary SM1 $(P<0.005)$ positively correlated with CPE.
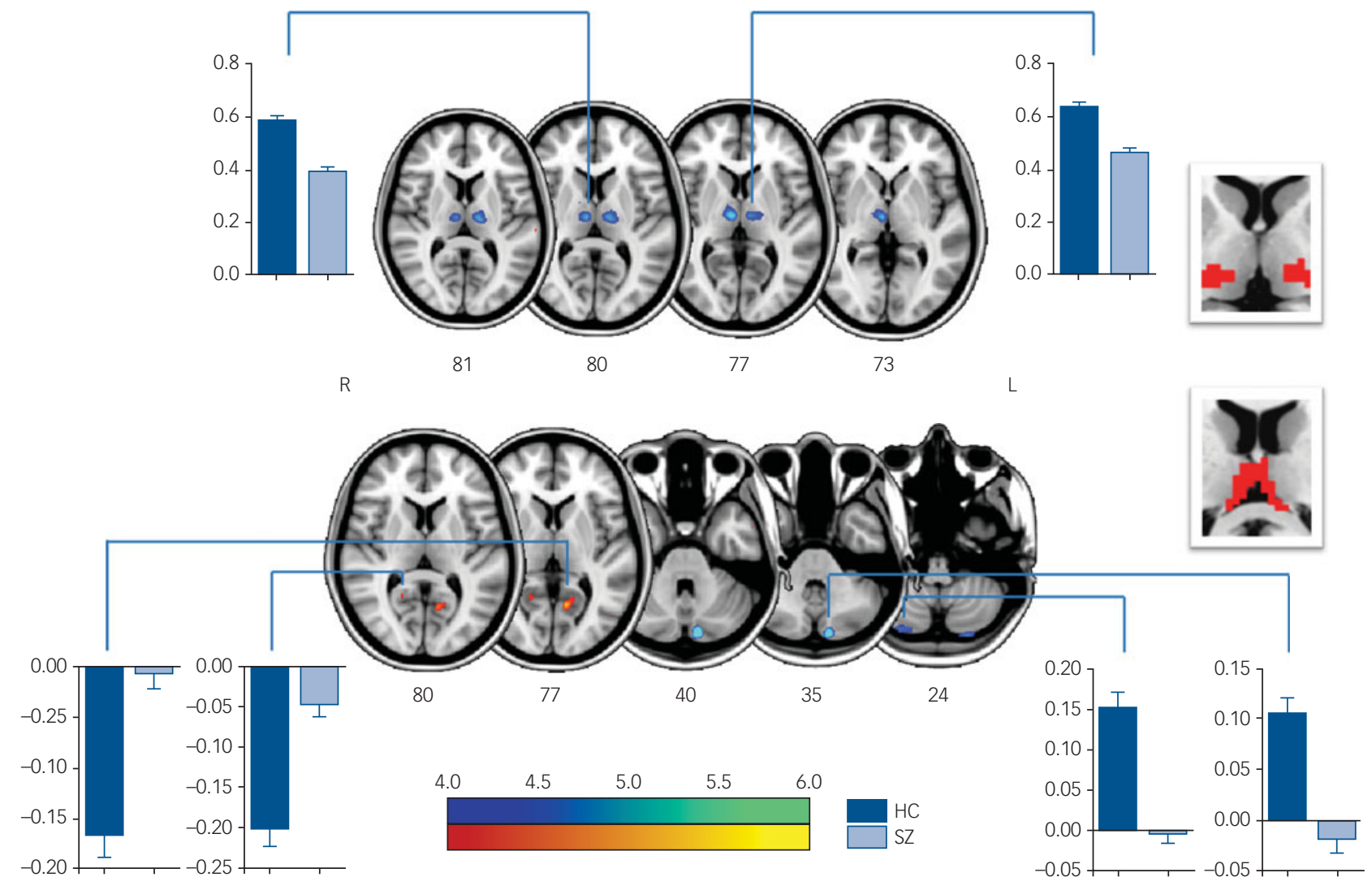

\section{Fig. 1 Significant intergroup differences in FC maps of thalamic subdivisions (ROI 2 and 3).}

The upper row shows that the functional integration of thalamic ROI 2 and other parts of thalamus was reduced in schizophrenic service users compared with healthy controls. The lower row shows that, compared with healthy controls, a decreased negative FC between thalamic ROI 3 and lingual gyrus exists, and a decreased positive FC between thalamic $\mathrm{ROI} 3$ and the left cerebellum were detected in patients with schizophrenia. In this case, it should be noted that the different regions shown in this figure were significant $p<0.05$ after FWE correction. The threshold of colour bar (indicating the $t$ value) is set for display.

$\mathrm{HC}$, healthy control group; L, left; R, right; SZ, schizophrenia group. 
(a)
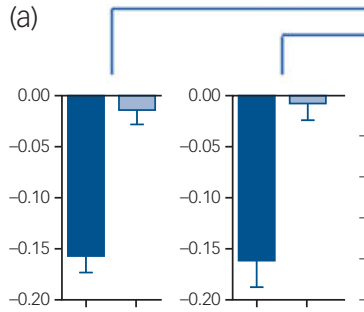

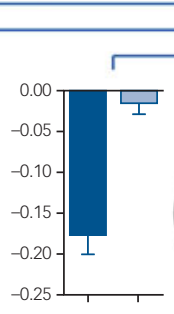

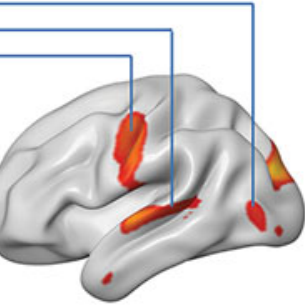
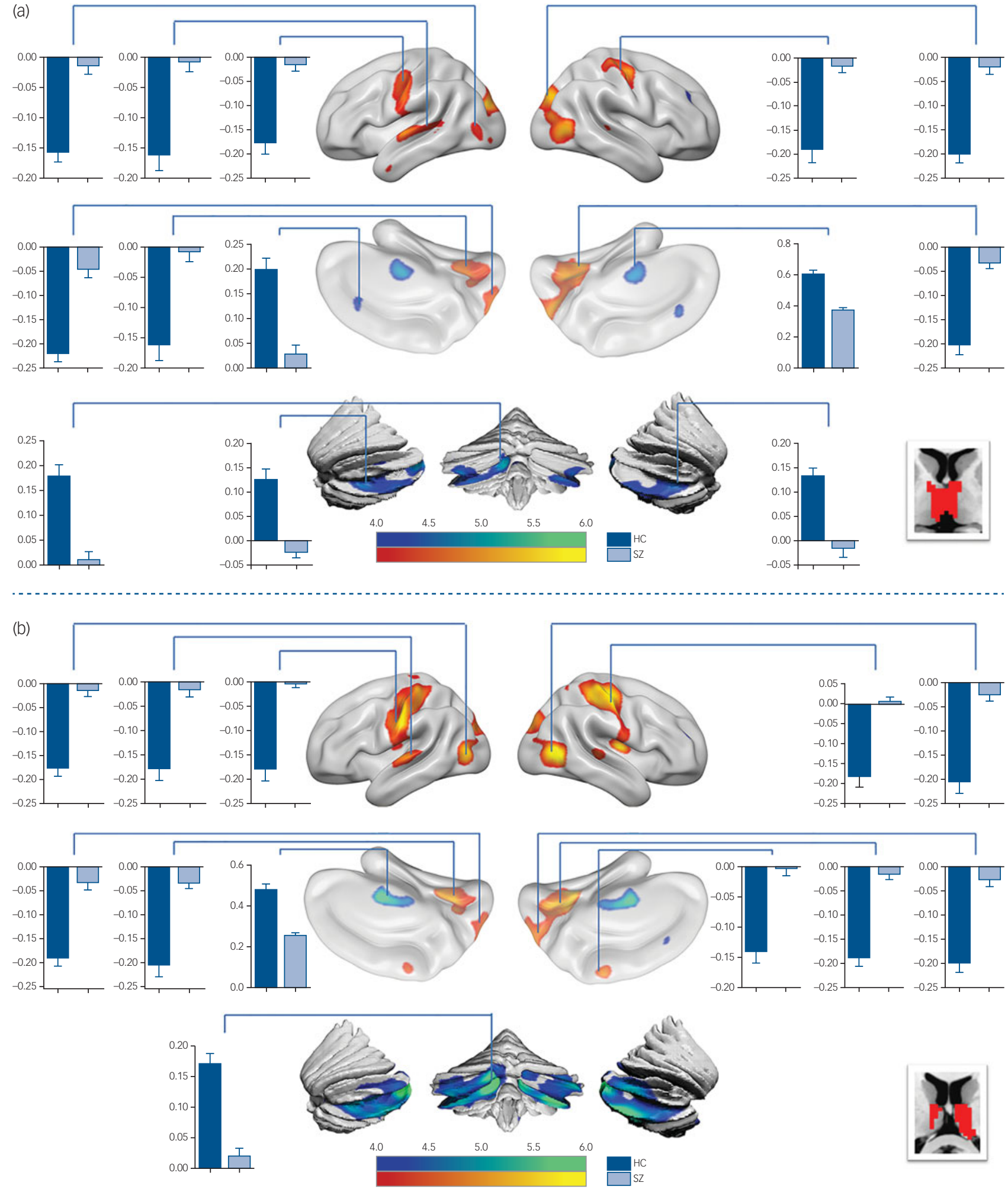

\section{Fig. 2 Functional connectivity comparison between the schizophrenia group and control group.}

(a) Negative FC of ROI 4 was found decreased in the schizophrenia group in sensorimotor network, visual cognition network and auditory cognition network, comparing with the HC group. Furthermore, a loss of positive interaction between ROI 4 and the left anterior cingulate cortex, as well as between ROI 4 and cerebellum, was observed in patients with schizophrenia. Additionally, the loss of intra-thalamic integration was detected in patients with schizophrenia. (b) The FC changes of ROI 5 in patients with schizophrenia is similar to that of ROI 4. Specifically, thalamic interaction with insula was found altered in patients. (c) Compared with $\mathrm{HC}$, FC of ROI 6 showed a decreased negative pattern in schizophrenia in sensorimotor network, visual cognition network, auditory cognition network and the posterior part of insula. In addition, a decreased positive interaction between ROI 6 and cerebellum was found in patients with schizophrenia. The loss of intra-thalamic integration was also detected in patients.

$\mathrm{HC}$, healthy control group; SZ, schizophrenia group. 

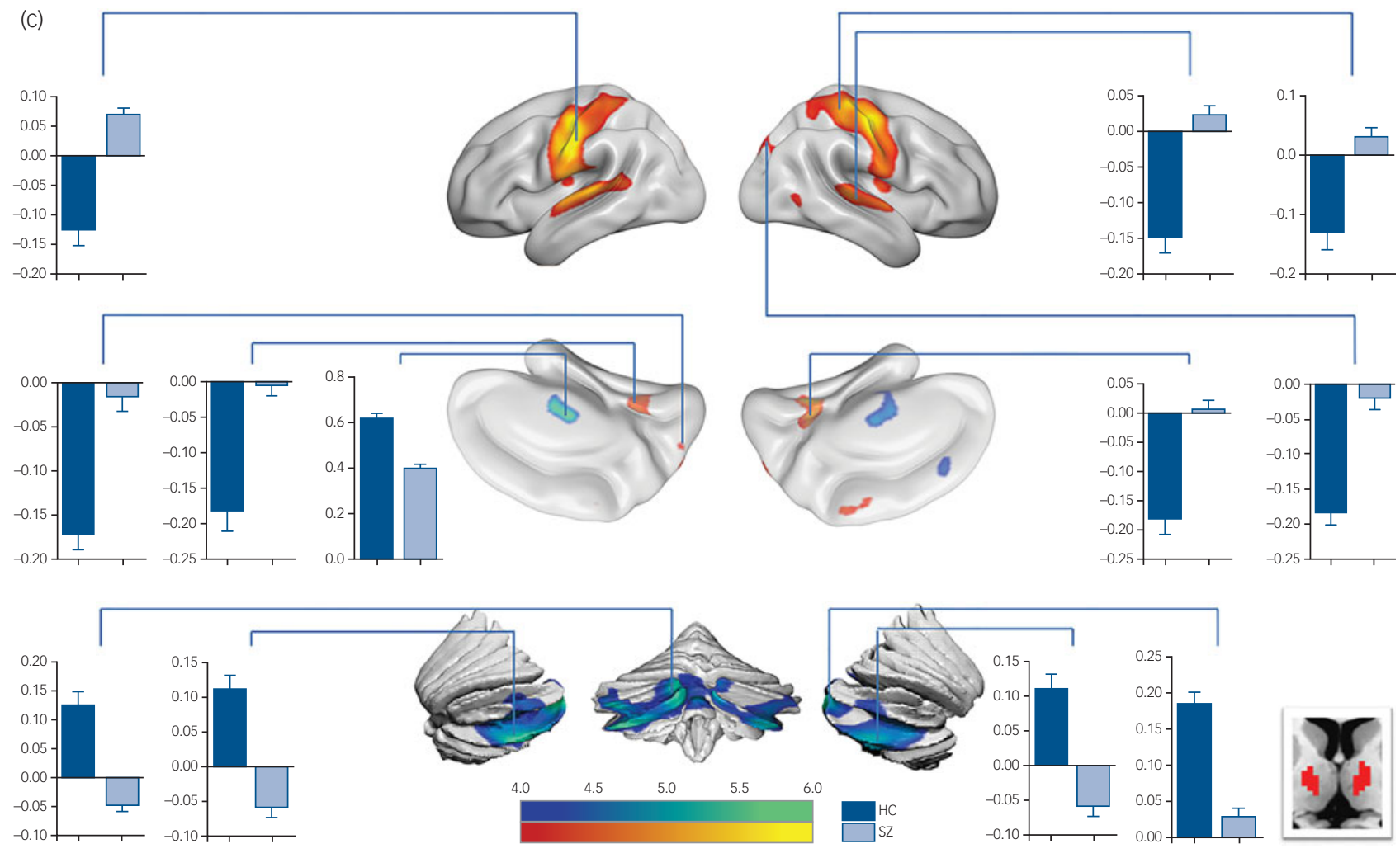

Fig. 2 (Continued)

\section{Discussion}

In this study, the thalamus was segmented using a data-driven approach based on the similarity of voxel-wise functional and structural connectivity. This method combined the advantage of the relatively high spatial resolution of dMRI, and the advantage of the high sensitivity of fMRI to long-range connectivity construction. In addition, another advantage of our method is that it does not require a priori information, and does not depend on a cortical atlas.

The thalamus was first segmented to six subdivisions in this study. Then, the functional connectivity analysis of each thalamic subdivision, especially in ventromedial, ventroanterior and dorsolateral subdivisions (ROI 4, 6, 5, respectively), demonstrated disturbances of the thalamo-cortical and thalamo-cerebellar connectivity in patients with schizophrenia. In particular, alterations of the thalamic interaction with perception and the motor system, and the disease effects on the thalamo-ACC interaction were found. In addition, loss of the thalamo-cerebellar connection was found gradually altered in the cerebellum (from anterior to posterior) with the order of thalamic subdivisions - (ventromedial (ROI 4) to ventroanterior (ROI 6) and then to dorsolateral (ROI 5) in patients. These findings reflected the altered functional connectivity of the cortico-cerebellar-thalamo-cortical circuit (CCTCC) in schizophrenia, suggesting more evidence in support of the disconnection hypothesis ${ }^{14-16}$ associated with cognitive abnormalities in schizophrenia. In addition, disrupted intrathalamic functional interaction in patients with schizophrenia was illustrated that may reflect an abnormality in the interaction between multiple systems regulated by the thalamus in schizophrenia.

In general, these results might imply that alterations in the functional coupling of the thalamo-cortical network in schizophrenia may be related to altered coactivity of multi nuclei within the thalamus rather than one specific nucleus (such as two higher order nuclei: the mediodorsal nucleus and the pulvinar). Additionally, this study may also provide new information: altered functional connectivity patterns of the thalamic subdivisions may help us understand the different aspects of the symptoms, such as sensorimotor symptoms, emotional symptoms and their relationship with the thalamus in people with schizophrenia.

\section{Thalamic dysconnectivity with perception and motor- related cortex}

Thalamic dysfunctions with perception and the motor-related cortex were found in the present study. Sensorimotor dysfunction in schizophrenia has been investigated by several previous studies. Our previous findings demonstrated decreased functional integration of the motor system in schizophrenia using local functional connectivity density that suggested motor-related symptoms, including a deficit of psychomotor and fine motor behaviour in schizophrenia. ${ }^{17}$ Recently, signal variability of the somatomotorrelated resting state networks (including the thalamus network) and the functional connectivity of these networks were found to be robustly impaired in schizophrenia. ${ }^{18}$ In line with previous studies, the decreased negative interaction between the somatomotor system and the thalamus in the schizophrenia group may reflect the abnormal somatosensory and motor behaviour of patients with schizophrenia.

Additionally, depending on the results of the data-driven parcellation, ROI 4, ROI 5 and ROI 6 cover two relay thalamic nuclei (first order nuclei): the ventral posterior nucleus and the ventral lateral nucleus. The former is part of the somatosensory system; the latter receives cerebellar input from the dentate nucleus and projects it to the primary motor cortex, part of the motor system. Thus, the alteration of perception and motor-related connections of these 


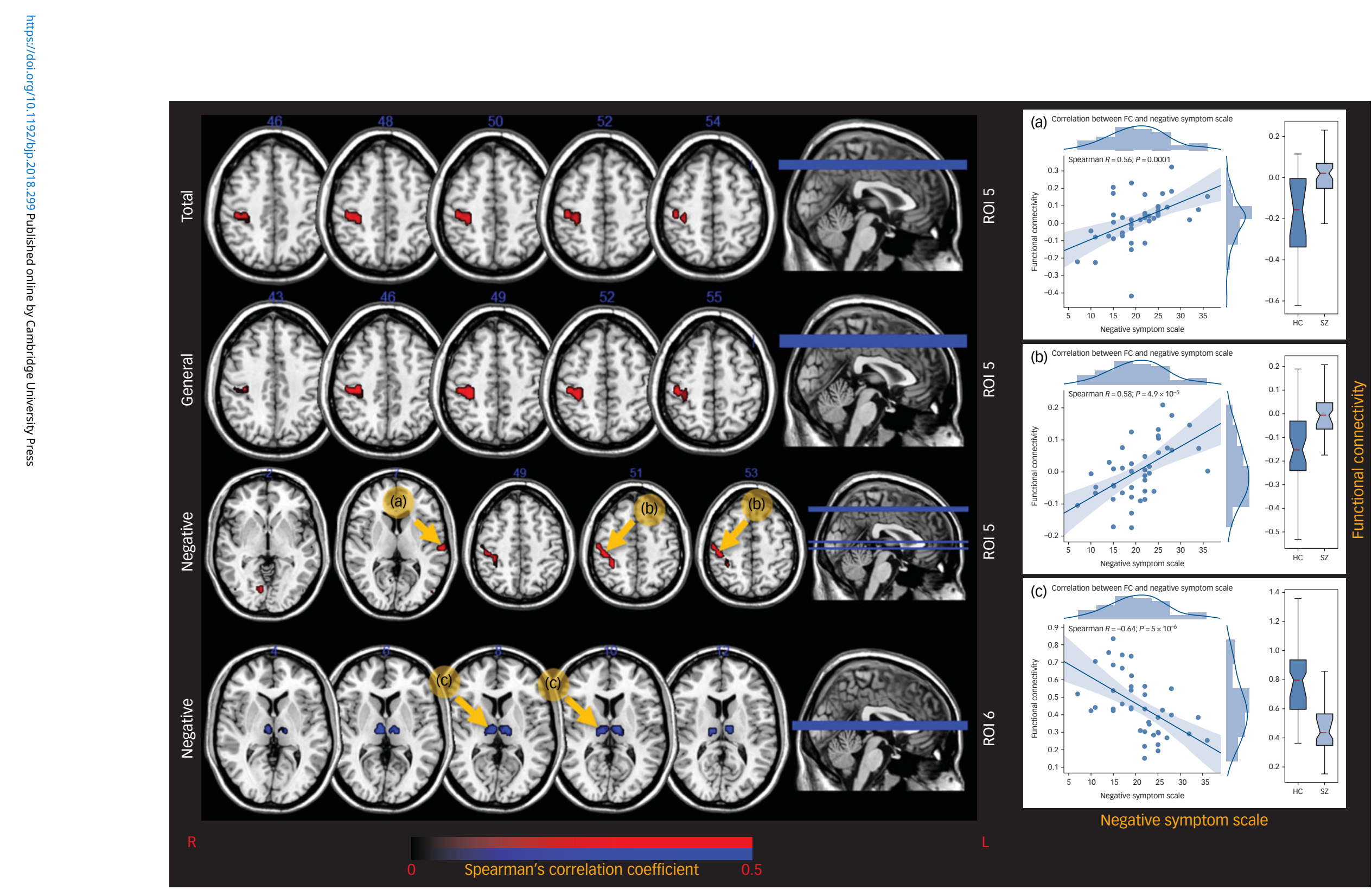

Fig. 3 The correlation maps between thalamic FC and PANSS scores.

Left panel demonstrates that the right precentral gyrus in the FC map of ROI 5 was positively correlated with total PANSS score (top, $p<0.001$, cluster size $>30$ voxels), general psychopathology scale (the second row, $p<0.001$, cluster size $>30$ voxels) and negative scale (the third row, $q<0.05$, FDR corrected, pointed by yellow arrow (b) in patients with schizophrenia. The FC of ROI 5 in the left superior temporal gyrus $(q<0.05$, FDR corrected, a) and the right lingual gyrus $(p<0.001$, cluster size $>30$ voxels) was also found

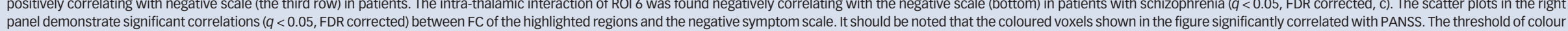
bar is set for display. 
three subdivisions in schizophrenia may suggest that the disease affects the ventral posterior and ventral lateral nuclei. This alteration in functional connectivity may provide a potential pathophysiological mechanism for perception-motor symptoms, such as dystonia, tremors, motor restlessness and hypokinesia in patients with schizophrenia. ${ }^{19}$

In our study, we observed loss of thalamic functional connectivity with the temporal lobe, the lingual gyrus, the cuneus and the precuneus in schizophrenia. Auditory and visual hallucinations are common clinical symptoms of schizophrenia. Accumulated evidence has suggested that the abnormality of the circuit in which the thalamus gate sensory input to the cerebral cortex contribute to the auditory and visual symptoms in patients with schizophrenia. ${ }^{20}$ Krause and colleagues provided evidence that the thalamic reticular nucleus was engaged in auditory gating and was disrupted in schizophrenia. ${ }^{21}$ The findings of Anticevic and colleagues that revealed a similar dysconnectivity pattern of the mediodorsal nucleus and lateral geniculate in schizophrenia, indicated that the thalamus and the cortex may be related to the abnormal visual function in schizophrenia. $^{22}$

In light of these previous studies, alterations in thalamic functional connectivity with the auditory and the visual cortex in the current study suggest that thalamo-cortical functional connectivity may be associated with aberrant auditory and visual processing in patients. In addition, altered thalamic subdivisions located at ROI 4-6, which cover the lateral posterior nucleus and the lateral dorsal nucleus anatomically connect with the visual cortical areas. Hence, an alteration would reflect the abnormal interaction between the visual system and the lateral dorsal/lateral posterior nuclei in patients. We speculate that abnormal thalamic subdivision functional connectivity with the auditory and visual cortex might contribute to hallucinations in schizophrenia. However, this hypothesis requires further confirmation in the future.

The posterior insula interacts with the somatosensory system and the ventral posterior inferior thalamic nuclei and involves functions such as multimodal sensory and pain processing. The increased functional connectivity between the thalamus and insula, consistent with a previous study, ${ }^{23}$ may reflect an abnormal information interaction, including sensory and self-awareness, between the thalamus and the insula.

Taken together, we found that the functional connectivity between the three thalamic subdivisions with SM1, the auditory/ visual cortex and the posterior insula in the schizophrenia group were different from those of the control group. Our results could reflect abnormalities of the integration of auditory/visual information that provides cues for motor function in schizophrenia and provide more evidence for the hypothesis that schizophrenia symptoms may arise from a cumulative cascade of impairments of the sensory system. In addition, we found that the precentral gyrus and the superior temporal gyrus lose negative interaction with the thalamus in schizophrenia; thus, the positive correlation between the functional connectivity of ROI 5 and the PANSS implied that the loss of the thalamic integration of motor information and auditory information was relevant to the patient's clinical symptoms.

\section{Altered thalamic-ACC pathway}

The ACC, which is involved in higher-level cognition functions such as attention, decision-making, monitoring response conflict and emotion is a brain region that is affected in schizophrenia. ${ }^{24}$ ACC dysfunction may be linked to the impaired ability to evaluate and learn from errors, social abnormality and emotional disorders in patients. Abnormal effective connectivity was found between the thalamus and the ACC in a recent study of patients with schizophrenia. ${ }^{25}$ Since the thalamus-ACC coupling is important for pain perception, ${ }^{26}$ our findings of decreased functional connectivity between the thalamus and the ACC may suggest a pathogenic mechanism of pain perception disorder in patients with schizophrenia.

\section{Loss of the thalamo-cerebellar connectivity}

In addition to abnormalities of the thalamo-cortical circuit, this study also illustrated a decrease in the functional connectivity of the thalamo-cerebellum in people with schizophrenia. The results are consistent with findings from previous studies. ${ }^{27}$ Emerging evidence suggests that the cerebellum is not only involved in motor function but also participates in a wide variety of cognitive processes by connecting with many cortical regions through the CCTCC. ${ }^{28}$ The altered functional connectivity of the CCTCC in schizophrenia found in the present study may provide more information to help us understand the impaired interaction among the thalamus, the cerebellum and the cortex in schizophrenia and its effects on abnormal cognition. ${ }^{29}$

Abnormalities in the Purkinje cell pathway, which modulates the activity of the cerebral cortex via deep nuclei, such as the dentate nucleus, and has an important role in gating information to the cerebral cortex in patients with schizophrenia has been shown. ${ }^{30}$ In addition, positive schizophrenia symptoms may be related to the failure in identifying internal and external events. ${ }^{31}$ Our results showed decreased functional connectivity in the thalamo-cerebellar network, and an enhancement of the interaction between the thalamus and the sensory cortex; and may perhaps provide neuroimaging evidence of imbalance of the cerebellar-thalamic inhibitory output in schizophrenia. This may be an important pathological cause of the positive symptoms of schizophrenia.

In addition, the posterior lobe and the vermis of the cerebellum showed decreased positive connectivity with the thalamus. The former, which is functionally connected with the prefrontal cortex and the posterior-parietal cortex, is involved in high-order processing for the integration of cognition and emotion. ${ }^{32}$ The vermis has been identified as the probable location of the limbic cerebellum for its functions in emotion and affection. ${ }^{33}$ The cerebellum participates in these non-motor functions by modulating the cortical areas corresponding to these functions via the thalamic association nuclei. The altered cerebellum-thalamic pathway and the thalamo-ACC connection in patients may share a similar pathological course in the thalamus. Furthermore, the thalamic ROIs 4, 5 and 6 are functionally negatively connected with the dorsal attention network in our control group. These results suggest the possibility that dysfunction of the cerebellum-thalamic functional connectivity could be the pathological mechanism of the frontal and the limbic symptoms, such as decreased emotion, decreased motivation and decreased attention in patients with schizophrenia.

\section{Decreased positive functional connectivity among the thalamic subdivisions}

Compared with the control group, deceased positive within-thalamic functional connectivity was found in the schizophrenia group. Results of the one-sample (demonstrated in supplementary Fig. 7) $t$-test revealed significant positive interconnections within the thalamus in both groups. This reflected that, although the thalamus is composed of multiple nuclei with diverse functions, interactions of these nuclei within the thalamus are primarily integrated. We hypothesise that the loss of integration within the thalamus may affect the multisystem interactions in schizophrenia. However, this speculation and the pattern of loss of the thalamic integration require further studies using ultra-high-resolution MRI data. 


\section{Correlation of thalamic functional connectivity and clinical features}

Intrathalamic integration of ROI 6 and functional connectivity of ROI 5 in the SM1 and the superior temporal gyrus, are associated with negative symptoms of patients with schizophrenia. These correlations reflect that the higher the patient's negative symptom score, the more change (compared with the control group) there is in thalamic functional connectivity. Previous studies have demonstrated that negative symptoms may be accompanied by abnormal involuntary movements or lack of movements. ${ }^{34} \mathrm{We}$ speculate that these results indicate that altered thalamic functional connectivity in the SM1 and loss of the thalamic integration may be related to abnormal motion control in patients with schizophrenia.

In addition, symptoms of patients may be related to abnormalities in the dopaminergic system. ${ }^{35}$ As the main output station of the basal ganglia, pallidal projection is located primarily in anterior and medial regions of the thalamus, where the ROI 5 and 6 covers. This suggests the possibility that the significant association between thalamic functional connectivity with schizophrenia symptoms may result from the influences of the abnormal basal ganglia dopamine system on thalamo-cortical connections.

In addition, the intrathalamic functional integration of ROI 4 and ROI 5 negatively correlated with the duration of disease, which may indicate that the functional integration of the thalamus would be progressively weakened with disease progression.

\section{Limitations and scope}

Several limitations of this study should be noted, including the potential circular logic of the method and the fact that the participants were mainly individuals with chronic schizophrenia (discussed in supplementary material). To address the first issue, we have carefully undertaken some supplementary calculations to support the results of this study (see supplementary Conjunction overlay). Regarding, the second issue, we will continue to collect data from patients both with first-episode schizophrenia ${ }^{36}$ and after interventions to investigate the role of the thalamo-cortical network in schizophrenia more thoroughly.

In addition, one of our results was only trend level: the higher the CPE was, the more thalamic functional connectivity in the cerebellum, the LOC and the SM1 changes in patients with schizophrenia. It is uncertain if this reflects the effect of drugs on abnormal functional connectivity. ${ }^{37}$ Since the dose of medication reflects severity of the disease, and relates to the functional connectivity changes in patients, this result may reflect the relation between the functional connectivity and the severity of the disease. This issue needs further investigations in future research.

In future, hypothesis-driven experimental research will be carried out and new methods ${ }^{38}$ will be used to seek the relationship between specific cognitive dysfunction and neuroimaging measurements in patients with schizophrenia so as to better understand the pathological features of schizophrenia.

\section{Implications}

This study used a data-driven approach to parcellate the thalamus by combining information about structural and functional brain connectivity. Pathways such as the superior-anterior thalamo-sensorimotor, the ventromedial thalamo-ACC and the superior-anterior thalamo-cerebellum were found altered in their functional connectivity when each thalamic subdivision was used as a seed. Meanwhile, intrathalamic integration was also found to be affected by the disease. The subdivisions that showed intergroup differences in functional connectivity mostly covered the mediodorsal nucleus and the ventral nuclei of the thalamus. Based on this parcellation and the functional connectivity results, the functional connectivity difference between the thalamus and the entire brain came from different nuclei of the thalamus. In general, the results of this study support the cognitive abnormalities seen in schizophrenia, further demonstrating a gradual pattern of functional connectivity alterations of the thalamic subdivision in the condition. Our results suggest that there is a local specificity in the alterations of the thalamus in patients, and this may increase our understanding of the relations between the CCTCC pathway and symptoms associated with schizophrenia.

Jinnan Gong (D), PhD Student, The Clinical Hospital of Chengdu Brain Science Institute, MOE Key Lab for Neuroinformation, Center for Information in Medicine, University of Electronic Science and Technology of China, P.R. China; and Visiting Scientist, McGill Centre for Integrative Neuroscience, Montreal Neurological Institute, McGill University, Canada; Cheng Luo, Associate Professor, the Clinical Hospital of Chengdu Brain Science Institute, MOE Key Lab for Neuroinformation, Center for Information in Medicine, University of Electronic Science and Technology of China, P.R. China; Xiangkui Li, Master Student, The Clinical Hospital of Chengdu Brain Science Institute, MOE Key Lab for Neuroinformation, Center for Information in Medicine, University of Electronic Science and Technology of China, P.R. China; Sisi Jiang, PhD Student, The Clinical Hospital of Chengdu Brain Science Institute, MOE Key Lab for Neuroinformation, Center for Information in Medicine, University of Electronic Science and Technology of China, P.R. China; Budhachandra S. Khundrakpam, Research Associate, McGill Centre for Integrative Neuroscience, Montreal Neurological Institute, McGill University, Canada; Mingjun Duan, Chief Physician, The Clinical Hospital of Chengdu Brain Science Institute, MOE Key Lab for Neuroinformation, Center for Information in Medicine, University of Electronic Science and Technology of China, P.R. China; Xi Chen, PhD Student, The Clinical Hospital of Chengdu Brain Science Institute, MOE Key Lab for Neuroinformation, Center for Information in Medicine, University of Electronic Science and Technology of China, P.R. China; Dezhong Yao, Professor, The Clinical Hospital of Chengdu Brain Science Institute, MOE Key Lab for Neuroinformation, Center for Information in Medicine, University of Electronic Science and Technology of China, P.R. China.

Correspondence: Cheng Luo, PhD, The Clinical Hospital of Chengdu Brain Science Institute, MOE Key Lab for Neuroinformation, Center for Information in Medicine, University of Electronic Science and Technology of China, Chengdu, China. Email: chengluo@uestc.edu.cn

First received 27 Feb 2018, final revision 5 Oct 2018, accepted 4 Nov 2018

\section{Funding}

This study was supported by the National Nature Science Foundation of China (No. 81771822 , 81330032, 81471638, 81861128001), Sichuan Province Science and Technology Support Project (No.2017SZO004, and 2017HH0001) and the Chinese Fundamental Research Funding for Central Universities in UESTC (ZYGX2016J121).

\section{Supplementary material}

Supplementary material is available online at https://doi.org/10.1192/bjp.2018.299.

\section{References}

1 Sherman SM. Thalamus plays a central role in ongoing cortical functioning. Nat Neurosci 2016; 19: 533-41.

2 Jiang $\mathrm{Y}$, Luo $\mathrm{C}$, Li X, Duan $\mathrm{M}, \mathrm{He} \mathrm{H}$, Chen $\mathrm{X}$, et al. Progressive reduction in gray matter in patients with schizophrenia assessed with MR imaging by using causal network analysis. Radiology 2018; 287: 633-42.

3 Cho KIK, Shenton ME, Kubicki M, Jung WH, Lee TY, Yun J-Y, et al. Altered thalamo-cortical white matter connectivity: probabilistic tractography study in clinical-high risk for psychosis and first-episode psychosis. Schizophr Bull 2016; 42: 723-31.

4 Lungu O, Barakat M, Laventure S, Debas K, Proulx S, Luck D, et al. The incidence and nature of cerebellar findings in schizophrenia: a quantitative review of fMR literature. Schizophr Bull 2013; 39: 797-806.

5 Craddock RC, Bellec $P$, Jbabdi S. Neuroimage special issue on brain segmentation and parcellation. Neurolmage 2018; 170: 1-4.

6 Woodward ND, Heckers S. Mapping thalamocortical functional connectivity in chronic and early stages of psychotic disorders. Biol Psychiatry 2016; 79: 1016-25.

7 O'Muircheartaigh J, Keller SS, Barker GJ, Richardson MP. White matter connectivity of the thalamus delineates the functional architecture of competing thalamocortical systems. Cereb Cortex 2015; 25: 4477-89. 
8 Jiang S, Luo C, Gong J, Peng R, Ma S, Tan S, et al. Aberrant thalamocortical connectivity in juvenile myoclonic epilepsy. Int J Neural Syst 2018; 28: 1750034

9 Gibbon M, Spitzer RL, Williams JBW, et al. Structured clinical interview for DSM-IV axis II personality disorders (SCID-II). American Psychiatric Press, 1997.

10 Kay SR, Fiszbein A, Opler LA. The positive and negative syndrome scale (PANSS) for schizophrenia. Schizophr Bull 1987; 13: 261-76.

11 Andreasen NC, Pressler M, Nopoulos P, Miller D, Ho B-C. Antipsychotic dose equivalents and dose-years: a standardized method for comparing exposure to different drugs. Biol Psychiatry 2010; 67: 255-62.

12 Haddock G, McCarron J, Tarrier N, et al. Scales to measure dimensions of hallucinations and delusions: the psychotic symptom rating scales (PSYRATS) Psychol Med 1999; 29: 879-89.

13 Frey BJ, Dueck D. Clustering by passing messages between data points Science 2007; 315: 972-6.

14 Friston KJ. Schizophrenia and the disconnection hypothesis. Acta Psychiatr Scand 1999; 99: 68-79.

15 Stephan KE, Friston KJ, Frith CD. Dysconnection in schizophrenia: from abnormal synaptic plasticity to failures of self-monitoring. Schizophr Bull 2009; 35: 509-27.

16 Dong D, Wang Y, Chang X, Luo C, Yao D. Dysfunction of large-scale brain networks in schizophrenia: a meta-analysis of resting-state functional connectivity. Schizophr Bull 2018; 44: 168-81.

17 Chen X, Duan M, Xie Q, Lai Y, Dong L, Cao W, et al. Functional disconnection between the visual cortex and the sensorimotor cortex suggests a potential mechanism for self-disorder in schizophrenia. Schizophr Res 2015; 166: 151-7

18 Kaufmann T, Skåtun KC, Alnæs D, Doan NT, Duff EP, Tønnesen S, et al. Disintegration of sensorimotor brain networks in schizophrenia. Schizophr Bull 2015; 41: 1326-35.

19 Walther S, Strik W. Motor Symptoms and Schizophrenia. Neuropsychobiology 2012; 66: 77-92.

20 Braff DL, Geyer MA, Light GA, Sprock J, Perry W, Cadenhead KS, et al. Impact of prepulse characteristics on the detection of sensorimotor gating deficits in schizophrenia. Schizophr Res 2001; 49: 171-8.

21 Krause M, Hoffmann WE, Hajós M. Auditory sensory gating in hippocampus and reticular thalamic neurons in anesthetized rats. Biol Psychiatry 2003; 53: 244-53.

22 Anticevic A, Yang G, Savic A, Murray JD, Cole MW, Repovs G, et al. Mediodorsa and visual thalamic connectivity differ in schizophrenia and bipolar disorder with and without psychosis history. Schizophr Bull 2014; 40: 1227-43.

23 Corradi-Dell'Acqua C, Tomelleri L, Bellani M, Rambaldelli G, Cerini R, Pozzi-Mucelli R, et al. Thalamic-insular dysconnectivity in schizophrenia: evidence from structural equation modeling. Hum Brain Mapp 2012; 33 740-52.
24 Polli FE, Barton JJS, Thakkar KN, Greve DN, Goff DC, Rauch SL, et al. Reduced error-related activation in two anterior cingulate circuits is related to impaired performance in schizophrenia. Brain 2008; 131: 971-86.

25 Wagner G, De la Cruz F, Schachtzabel C, Güllmar D, Schultz CC, Schlösser RG, et al. Structural and functional dysconnectivity of the fronto-thalamic system in schizophrenia: a DCM-DTI study. Cortex 2015; 66: 35-45.

26 Yen C-T, Lu P-L. Thalamus and pain. Acta Anaesthesiol Taiwan 2013; 51: 73-80.

27 Mittal VA, Dean DJ, Bernard JA, Orr JM, Pelletier-Baldelli A, Carol EE, et al. Neurological soft signs predict abnormal cerebellar-thalamic tract development and negative symptoms in adolescents at high risk for psychosis: a longitudinal perspective. Schizophr Bull 2014; 40: 1204-15.

28 Middleton FA, Strick PL. Cerebellar projections to the prefrontal cortex of the primate. J Neurosci 2001; 21: 700-12.

29 Andreasen NC, Paradiso S, O'Leary DS. 'Cognitive dysmetria' as an integrative theory of schizophrenia: a dysfunction in cortical-subcortical-cerebellar circuitry? Schizophr Bull 1998; 24: 203-18.

30 Reyes M, Gordon A. Cerebellar vermis in schizophrenia. Lancet 1981; $\mathbf{3 1 8}$ 700-1.

31 Simons CJP, Tracy DK, Sanghera KK, O'Daly O, Gilleen J, Dominguez M-G, et al. Functional magnetic resonance imaging of inner speech in schizophrenia. Biol Psychiatry 2010; 67: 232-7.

32 O'Reilly JX, Beckmann CF, Tomassini V, Ramnani N, Johansen-Berg H. Distinct and overlapping functional zones in the cerebellum defined by resting state functional connectivity. Cereb Cortex 2010; 20: 953-65.

33 Stoodley CJ, Valera EM, Schmahmann JD. Functional topography of the cerebellum for motor and cognitive tasks: an fMRI study. Neurolmage 2012; 59: 1560-70.

34 Whitty PF, Owoeye O, Waddington JL. Neurological signs and involuntary movements in schizophrenia: intrinsic to and informative on systems pathobiology. Schizophr Bull 2009; 35: 415-24.

35 Crow TJ. Positive and negative schizophrenic symptoms and the role of dopamine. Br J Psychiatry 1980; 137: 383-6.

$36 \mathrm{He} \mathrm{H}$, Yang M, Duan M, Chen X, Lai Y, Xia Y, et al. Music intervention leads to increased insular connectivity and improved clinical symptoms in schizophrenia. Front Neurosci 2018; 11: 744.

37 Kraguljac NV, White DM, Hadley N, Hadley JA, ver Hoef L, Davis E, et al. Aberrant hippocampal connectivity in unmedicated patients with schizophrenia and effects of antipsychotic medication: a longitudinal resting state functional MRI study. Schizophr Bull 2016; 42: 1046-55.

38 Jiang Y, Luo C, Li X, Li Y, Yang H, Li J, et al. White-matter functional networks changes in patients with schizophrenia. Neurolmage 2018; April 13 (Epub ahead of print). 